Mots. Les langages du politique

$81 \mid 2006$

Suisse, laboratoire politique européen ?

Jacques Montredon, De bouche à oreille. Dictionnaire des étudiants étrangers de Besançon

Maurice Tournier

\title{
OpenEdition
}

Journals

Édition électronique

URL : https://journals.openedition.org/mots/738

DOI : $10.4000 /$ mots.738

ISSN : 1960-6001

Éditeur

ENS Éditions

Édition imprimée

Date de publication : 1 juillet 2006

Pagination : 129-131

ISBN : 2-84788-098-4

ISSN : 0243-6450

Référence électronique

Maurice Tournier, « Jacques Montredon, De bouche à oreille. Dictionnaire des étudiants étrangers de Besançon ", Mots. Les langages du politique [En ligne], 81 | 2006, mis en ligne le 01 juillet 2008, consulté le 23 avril 2022. URL : http://journals.openedition.org/mots/738 ; DOI : https://doi.org/10.4000/mots. 738

(C) ENS Éditions 
La lecture critique que propose J. Butler des deux auteurs isole ici le concept de «réinscription » proposé par J. Derrida (p. 226) : c'est en effet dans la rupture entre le signe et ses usages, entre le performatif et son contexte que git la promesse de «resignification subversive » (p. 244) qui peut permettre aux victimes des discours de haine de les «vider de leur charge d'humiliation » et de se réapproprier leur force, au profit d'un « discours insurrectionnel».

Au terme de ces analyses remarquables de densité et de puissance critique, le lecteur français, s'il est familier des travaux d'Évelyne Larguèche sur l'injure, situés eux aussi au confluent de la linguistique et de la psychanalyse, regrettera peut-être qu'en centrant l'analyse sur la performativité, J. Butler tende à réduire les discours de haine à des adresses injurieuses, gommant par là la présence du «tiers », tantôt témoin, tantôt destinataire direct ou indirect du discours injurieux, et aplatissant ainsi le triangle constitué par l'injurieur, l'injurié et le « tiers » qui constitue pour É. Larguèche l'une des clés de l'efficace de l'injure.

En revanche, les analyses de J. Butler, inscrites dans le contexte des débats juridiques et des problématiques institutionnelles propres aux États-Unis, sont loin de constituer une " affaire américaine », comme le souligne très justement sa traductrice (p. 17) : parité, harcèlement, pornographie, foulard islamique et homophobie lui fournissent autant d'exemples de thèmes qui exigent, selon C. Nordmann, de « déterritorialiser Butler».

Claire Oger

Université Paris 13-Paris Nord, Céditec

claire.oger@club-internet.fr

\section{De bouche à oreille. Dictionnaire des étudiants étrangers de Besançon} Jacques Montredon, préface de Bernard Py 2005, Besançon, Cêtre, $271 \mathrm{p}$.

Comment les mots et les expressions résonnent-ils en nous? Qu'est-ce qu'ils évoquent de leurs fonctions, de leurs référents, de leurs valeurs? Pour en avoir une idée, interrogeons des témoins auditeurs, et les plus éloignés de notre langue au départ puisqu'il s'agit d'étrangers en situation d'apprenants. Une telle enquête, scrupuleusement conduite par Jacques Montredon, a été menée tout au long de huit années d'entretiens et d'annotations au Centre de linguistique appliquée de Besançon. Ces étudiants se sont efforcés de répondre à leur enseignant, hors toute normativité, en se posant la question : "Qu'est-ce que j'ai compris dans les emplois de tel ou tel signe vocal que j'ai rencontré? »

Il s'en est suivi un ouvrage qui présente, dans l'ordre alphabétique du touche-à-tout habituel au dictionnaire, une série non de définitions mais de récits 
d'interactions et de remarques commentées faisant effort pour arriver à un sens. On sait que ce dernier n'est pas à presser comme un citron dont on extrait le jus, mais à humer parmi les odeurs, à gouter parmi les saveurs, à recoudre sans cesse avec les fils des situations et de l'expérience acquise... Images que tout cela, bien entendu, puisqu'il s'agit, en fait, de culture. "L'apprenant est un ethnographe », disait C. Roberts.

L'attestation d'emploi et l'analyse de sa réception sont ici reines. Enfin un dictionnaire de signes linguistiques qui part de témoignages et du discours compris, ou plutôt ressenti ! Chaque exemple est saisi sur le vif, dans sa simplicité native, parfois sa nouveauté et la surprise qui l'accompagnait (pour prendre les deux premières rubriques, l'étudiant se demandait ce que signifient être achesse ou Vas-y, accouche! ; une étudiante s'avoue "perdue » devant l'énoncé : C'est pas terrible). Jolie quête, pleine de savoureuses trouvailles, que peut teinter une légère pointe d'humour. Le tri du bon usage n'est volontairement pas fait: on se promène dans le familier, les modes, le populaire, le verlan, voire l'argot, bref tout ce qui émaille notre discours bien français de tous les jours d'aujourd'hui (envoyer balader, cartonner, cramer, en faire un fromage, ça me gonfle, voilà le hic, keuf, mince !, c'est nickel, y a pas photo, pioncer, faire le pont, quand même, pas de quoi, être scié, sécher, et toc!, si tu veux, 68... ). Mais l'intérêt du livre ne s'arrête pas à une récolte d'anecdotes.

Une entame revient souvent: "Dans le contexte, j'ai compris... », « J'ai appris par la situation... », «En m'expliquant la situation, elle a dit... » On trouve en effet, à chaque rubrique, la priorité que l'auditeur à la recherche du sens confère spontanément au contexte (discursif, élocutif, situationnel, désignatif, comportemental, imaginaire...). Il prête attention à :

- l'entourage immédiat du mot; «Elle a dit "Ça cramait! Il y avait de la fumée partout" ";

- son intonation; à propos de Purée !: «On dit ça avec la même intonation que Misère ! »;

- l'évènement; «Mon amie m'expliquait qu'elle venait d'attendre plus d'une heure en vain [...]. Une Française qui était avec nous a constaté : “Il t’a posé un lapin !" J'ai compris... »;

- un mouvement désignatif ; à propos de voiture-balai : «Il indiquait l'entrée du métro... »;

- la mimique; concernant navet et peut-être ça craint ! : «Par la mine du jeune qui a prononcé cette phrase, j’ai compris que ce film était nul »;

- la gestuelle ; "Il m'a expliqué en disant: “il lève bien le coude” et il a fait le geste de lever un verre à la bouche »; tout en disant récupérer, un ami « a fait le geste de ramasser »;

- l'analogie attachée au mot-image (panier percé, parler cash, se scotcher... «c'est très visuel »).

Ce qui n'empêche pas l'apprenant de faire parfois fausse route, de chercher 
plus loin, y compris dans les dictionnaires «de langue » - ce qui peut l'amener à constater « les décalages entre langue enseignée et langue rencontrée » (Bernard Py).

La préface de B. Py insiste à juste titre sur l'intérêt pédagogique de cette sorte d'enquête, plaidant en faveur des méthodes communicatives. Elle permet à l'apprenant, mais aussi à l'enseignant, de se méfier des pièges, faux amis, àpeu-près et malentendus, louanges négatives ou péjorations positives - confusion de l'anglais preservative et du français "préservatif », rapprochement trop hâtif (chantier interdit ne signifie pas « interdit de chanter »), compliment humoristique (c'est du beau! / du propre!) ou péjoration élogieuse (c'est une bombe!, gueule d'amour); elle leur permet de prêter attention à la différence de génération des locuteurs (ainsi pour sortir avec) ou, à l'inverse, de s'appuyer sur des équivalences au sein de la même langue (dans un site d'emploi identique, un apprenant rapproche «Qu'est-ce que c'est que cette salade!», «Qu'est-ce que c'est que ce bordel!» et «Qu'est-ce que c'est que cette histoire! », tout en soupçonnant des registres de niveaux différents) et entre deux langues dont l'une fait référence pour l'apprenant - à propos des « cheveux qui se dressent sur la tête ", un Anglais évoque : "It made my hair stand on end » (un travail sur les différences culturelles peut être esquissé à partir de là, puisqu'il s'agit en anglais des poils du cou); un autre traduit « avoir un chat dans la gorge » par " to have a frog in one's throat», le chat étant anglais et la grenouille française, comme il se doit...

Tous ces exemples n'amorcent, en fait, qu'un début d'enquête. Leur répertoire alerte sur de multiples problèmes de compréhension et de traduction et reste ouvert à toute continuation, à l'infini des expériences langagières, puisqu'une langue «vivante » est une création continuée. Ni dictionnaire d'expressions orales, ni manuel pédagogique, ce bijou de (trop) petit livre débouche sur une didactique du dialogue-recherche entre enseignant et étudiant en quête de consensus sur la signification, sans trop présupposer que l'exégèse de la parole écoutée soit l'apanage du seul enseignant institué en propriétaire de la norme. Ya-t-il d'ailleurs une norme du sens?

Maurice Tournier

Directeur de recherches émérite au CNRS, maurice.tournier@wanadoo.fr 